\title{
CIENCIA Y TEORÍA CURRICULAR
}

\author{
DARós, W. R. \\ Amanábar 1238. 2000 Rosario. Argentina.
}

\section{SUMMARY}

The purpose of this study is to pay attention to the structure of scientific thinking as a means to form the curriculum. Therefore an analogic concept of science is analysed in order to be useful to different kinds of knowledge and academic matters.

This study implies that science thought begins in everyday's thought perfected. In this way science is a powerful tool to develop the human will and moral conduct of a person. The scientific thought is particularly efficient for a didactic theory to balance the psychological aspect of mind in the students with the logic and objective demands.

\section{INTRODUCCIÓN}

El presente trabajo se propone como objetivo acentuar la importancia que tiene la estructura de un proceder científico en el momento de elaborar un instrumento didáctico como es el currículo. Para esto es necesario clarificar el concepto analógico de ese proceder, de modo que pueda ser útil para diversos saberes específicos, incluidos algunos aspectos de las humanidades.

El trabajo parte del supuesto de que las ciencias y sus procederes, con sus grandezas y limitaciones, son construcciones humanas, que prolongan y perfeccionan el proceder cotidiano. Las diversas ciencias así concebidas poseen un magnífico potencial para hacer desarrollar y crecer al ser humano, no sólo en el aspecto intelectual, sino también en el imaginativo, volitivo y moral, contribuyendo a generar lo que llamamos la persona y los valores humanos.

Las ciencias, en particular, constituyen una excelente base que los docentes deberían tener en cuenta equilibrando el peso de currículos que, al estar centrados únicamente en la variable psicológica, social o política de las personas, minusvaloran la racionalidad del proceder científico.
Teniendo en cuenta el objetivo del presente trabajo, trataremos los siguientes puntos:

a) En primer Iugar, el concepto de ciencia, haciendo notar que en la historia de nuestra cultura occidental la ciencia no constituye un concepto unívoco ni equívoco sino análogo, por to que el concepto de conocimiento científico puede ser insertado útil y legítimamente en diversos tipos de saberes actuales.

b) A pesar de la amplitud de aplicación que puede recibir el concepto de ciencia como instrumento educativo, éste posee valores y límites humanos que deben ser valorados y reconocidos (como el valor gnoseológico y epistemoÍgico de búsqueda de la objetividad científica, el valor moral del amor a la verdad, el respeto y la precaución por las consecuencias de las acciones que se derivan de las invenciones científicas).

c) En tercer lugar, trataremos de la incidencia de los valores y límites de la ciencia en un modelo curricular, indicando primeramente Ia finalidad que poseen las instituciones educativas escolares y que no poseen, por ejemplo, las instituciones familiares aun cuando ellas 
son agentes primarios del proceso educativo humano. Resaltaremos entonces el valor de los pasos sistematizadores del proceder científico, sin minusvalorar la riqueza intuitiva y afectiva del proceder espontáneo, cotidiano y familiar. Se acentuará, pues, desde la perspectiva del proceder científico el valor de la reflexión sístemática sobre el propio proceso de aprender.

d) Se confrontarán, con un deseo integrador, los objetivos del conocer y del proceder en el ámbito cotidiano, en el ámbito científico y en el ámbito didáctico, acentuándose la jodea de que el docente, en una teoría didáctica, debería tener presente tanto los conocimientos previos de quien aprende, como la base epistemológica de los conocimientos que intenta hacer construir en la situación de una didáctica científica. Un modelo curricular, entendido como instrumento didáctico, debería contener tanto una base epistemológica clara y actualizada de los saberes científicos socialmente admitidos por la comunidad científica, como una concepción de las posibilidades psicológicas de los que aprenden, de modo que fuese posible pensar la acción de los docentes como puente facilitador y constructor de los saberes con sus aprendices.

Los argumentos que se aportan para apoyar la propuesta del trabajo se basan en la estructura logica y epistemológica, insita en el concepto análogo de ciencia. Esta estructura complementa la riqueza de los valores de Ia vida cotidiana, exigiendo un esfuerzo explficito de tendencia a la objetivación, sistematización y validación social o intersubjetiva no sólo de los conocimientos sino también de las conductas humanas, sin que por ello se ignore la riqueza humana de la creatividad, de la vida afectiva porque también desde el punto de vista científicoel hombre es afectado; pero actúa (como ideal ćtico al cual tienden las instituctones educativas) o debería actuar con un proceder más creativo y racional en el ámbito del conocimiento, y más universal y justo en el ámbito de la ética. I) este modo, se argumenta que es posible gencrar una forma de ser (una formación) más integrada que aquélla que se guía por otros modelos curriculares, los cuales acentúan y priman, con cierto desequilibrio, ya lo afectivo, ya lo racional, ya lo creativo, ya lo social. Otro argumento en favor de esta propuesta se halla en el hecho de que no es necesario rentnciar al avance científico y tecnológico para recuperar algunos valores típicamente humanos, que algunos pedagogos ven amenazados al interpretar el método científico de una manera reduccionista y no analógica e inserta en el ser humano, sin ignorar su cotidianeidad, y su condición psicológica, como aparece en la presente propuesta.

Otra razón que se aporta para sostener la viabilidad de la propuestá se halla en la idea de que la ciencia es una construcción humana, realizada por hombres y lo que los hombres realizan también afecta a los hombres por una implicación dialéctica, donde el sujeto crea el objeto, pero éste revierte sobre el sujeto, generándose frecuentemente entre la estructura del sujeto y aquélla del objeto creado una interestructuracion creciente. El producir un sistema racional, como es una ciencia, el utilizarlo y trabajar con él, condiciona al hombre dándole posibili- dades para ser más racional, aunque no lo determina, generando en él necesariamente un comportamicnto racional en otros ámbitos de la vida humana. En este sentido, las instituciones escolares pueden generar, a través de un currículo de base epistemológica, condiciones de posibilidad, pero no determinan (ni deberian determinar para ser éticas) la conducta humana, porcue el proceder científico, al mismo tiempo que cxige lógica y prueba, exige también libertad, creatividad y sentido de la finalidad de las acciones en un contex to humano y social.

\section{EL CONCEPTO DE CIENCIA}

Si tenemos en cuenta nuestra cultura occidental, no resulta fácil establecer a qué nos referimos con ol tćrmino de ciencia. Sabemos que para Aristóteles la ciencia era una forma de saber que partiendo de premisas verdaderas y utilizando el silogismo epistemológico llegaba a conclusiones también verdaderas'. Por el contrario, después del Renacimiento, la ciencia implica partir de hechos problemáticos que exigen nuevas hipótesis, las cuales requieren validación y permiten obscrvar nuevos hechos o reinterpretarlos de forma más eficiente, económica y armónica.

El hombre siempre necesitó recurrir a formas de saber que consideraba válidas en su época. Primeramente el saber mítico de los ancianos, Juego el saber revelado en Jibros sagrados (Vedas, Biblia, Corán) o en personas que adquirieron gran prestigio (Platón, Aristótcles).

Mas lenta pero inexorablemente los hombres fueron exigiendo que los conocimientos se validasen no por la fuente de la cual se asumen, sino por la forma de justificar las afirmaciones.

De hecho, la ciencia heredó el prestigio del saber propio, en otros tiempos, del saber religioso.

Es sabido que, tras el prestigio que ha logrado en la modernidad el modo de proceder de la ciencia, no pocos cientificos de un sector (química, física, biología, matemáticas) han descado monopolizar el concepto de ciencia, esto es, hacerlo unívoco, de modo que aquello que no se adecua a este modo de proceder que ellos ejercían no podia considerarse científico.

En este contexto, otros autores han llegado a considerar que el concepto de ciencia es equívoco: todo vale. Todo conocimiento en principio es humano y no hay un criterio para establecer un único concepto de ciencia?

Finalmente, una epistemología que tiene en cuenta la historia del concepto de ciencia en occidente estima que lo más adecuado es considerar que ese concepto no es ni unívoco ni equívoco, sino análogo. No existe sólo la ciencia, una única forma de considerar los conocimientos como científicos; ni tampoco existen solamente diferentes ciencias (las ciencias, en plural) sin ningún elemento común por el que se las pueda calificar como tales ${ }^{3}$. 
El término ciencia es análogo, como la mayoría de nuestros conceptos. EI concepto hombre, mesa, árbol, indica algunos elementos comunes a los hombres, las mesas y los árboles concretos y singulares que son siempre en parte diversos. Al hablar de la ciencia deberíamos, pues, buscar el o los elementos comunes a todas las ciencias particulares.

Ciertamente siempre habrá algo de convencional en cómo queremos llamar a las cosas, pero debemos establecer el criterio que empleamos para designarlas. Pues bien, cuando queremos establecer a qué tlamaremos ciencia, algunos se atienen solamente a lo que hoy se considera ciencia empírica, pero ignoran la historia de occidente sobre este concepto y los criterios con los cuales en otros tiempos se llamó ciencia a la ciencia.

«Siempre será un asunto a resolver por una convención o una decisión -afirma Popper- a qué cosa hemos de llamar una ciencia o a qué hemos de calificar de cientí ficons.

«Las ciencias, después de todo, son nuestra propia creación -según Feyerabend- incluidos todos los severos estandars que parecen imponernos. Es bueno recordar constantemente el hecho de que es posible escapar de la ciencia tal como la conocemos"s.

La creciente «especialización -ha escrito A. Einsteinhace cada vez más difícil que podamos captar de modo general la ciencia en su conjunto, sin lo cual el verdadero espíritu de investigación queda mermado sin remedio, a medida que aumenta el progreso científico ${ }^{6}$.

El proceso científico que produce la ciencia comparte, con el conocimiento cotidiano, el interés por investigar (in vestigia ire: seguir las huellas, de manera que de los efectos, fenómenos o apariencias se llegue a las causas que las justifican). Pero la ciencia añade, al modo cotidiano de conocer, la precisión (conceptual, linguiística y metodológica) de manera que las hipótesis y teorías, libremente inventadas, queden justificadas con algún recurso públicamente constatable.

La ciencia, pues, en general, y cada ciencia en particular implican un doble proceso: a) de investigación inventiva mediante un sistema teórico, y b) de precisa justificación crítica respecto de los variados objetos que estudia.

En este sentido, y referido a la física teórica, afirmaba Einstein:

«La ciencia es el intento de lograr que la diversidad caótica de nuestras experiencias sensoriales corresponda a un sistema de pensamiento lógicamente uniforme. En este sistema, cada experiencia debe estar en correlación con la estructura teórica, de tal modo que la relación resultante sea única y convincente.

"Las experiencias sensoriales representan lo dado. Pero la teoría que tendrá que interpretarlas está hecha por el hombre. Se trata del resultado de un proceso de adaptación de carácter extremadamente arduo: hipo- tético, nunca definitivo, siempre sujeto a la crítica y a la duda.

"La manera científica de formar conceptos se distingue de la que utilizamos en la vida de cada día no substancialmente sino sólo en la mayor precisión de las definiciones de los conceptos y las conclusiones, una acción más esmerada y sistemática del material experimental, una mayor economía lógica. Esto último significa el esfuerzo por reducir todos los conceptos y correlaciones a la menor cantidad posible de conceptos y axiomas básicos lógicamente independientes $)^{7}$.

La ciencia, pues, en general y cada ciencia en particular, implica un sistema de pensamientos lógicamente unifor$m e$. La ciencia es una forma de pensar que o es lógica o requiere de la lógica. Tanto la matemática como la física - la ciencia de la educación requieren la creación de hipótesis (que son pura invención humana) sometidas a la coherencia lógica. La lógica significa lo mínimo para que exista una justificación científica en el modo de proceder científico.

Ahora bien cuando una ciencia pretende hacer hipótesis o interpretaciones sobre la realidad empirica (física, histórica, educativa, etc.), las hipótesis deben estar justificadas por un correlato con la realidad empírica a la cual se refieren. Deben estar justificadas por nuevos hechos, datos, fenómenos que confirmen (o hagan más firme) la persuasión, siempre precaria en ciencia, de que las interpretaciones han sido confirmadas por nuevos hechos. Estaríamos encerrados en un círculo vicioso si nos declarásemos contentos con los hechos interpretados por la teoría que hemos inventado y, a su vez, declarásemos verdadera la teoría porque es avalada por los hechos para los cuales hemos inventado ese teoría. Si para explicar por qué se encrespa el mar hacemos la hipótesis de que Neptuno está erojado y mueve las aguas con su tridente, no podemos decirle a quien nos pregunta si es verdad tal interpretación: «iCaramba, mire cómo está encrespado el mar!». Un hecho, en efecto, puede ser interpretado por diversas teorías sin que por esto el hecho las confirme como verdaderas. El único elemento de juicio en pro de lo que explica no puede ser lo que debe ser explicado ${ }^{8}$.

Es justamente este recurso a la realidad empírica lo que distingue a las ciencias empíricas de las formales y de la literatura (novela, teatro, poesía, etc.). La literatura puede referirse a la realidad (por ejemplo, a la realidad psicológica), puede inventar interpretaciones originales, referidas a hechos reales, sobre los problemas humanos, pero no pretende ofrecer medios de confirmacion de sus afirmaciones. Mas la literatura comparte con las ciencias un proceso creador, imaginativo, inventor de mundos con significados. La ciencia lo hace con sus hipótesis; la Iiteratura, con sus interpretaciones o creaciones poéticas'.

«Insistir en la diferencia entre la ciencia y las humanidades ha sido, durante largo tiempo, una moda y se ha 
convertido en una pesadez: ambas practican el método de la resolución de problemas, el de las conjeturas y las refutaciones; Io mismo se practica en la reconstrucción de un texto corrupto que para construir una teoría de la radioactividad» ${ }^{10}$.

Es ese recurso a la realidad empírica lo que hace de la ciencia empírica algo relativo a su objeto, objeto siempre limitado y observable al menos en sus efectos. Pero sería un abuso reducir toda ciencia a los cánones de la ciencia empírica y negar como no humanamente importante, o de ninguna manera válido, la asunción de valores vitales absolutos o metafísicos que suelen aparecer en los escritos literarios". También sería un abuso reducir toda la objetividad de los pensamientos a un referente empírico, como si la no contradicción lógica no fuese un elemento esencial de la objetividad humana ${ }^{12}$.

\section{VALORES Y LÍMITES HUMANOS DE LAS CIENCIAS}

El proceder científico que produce la ciencia en general y las ciencias en particular, es una cuestión de inteligencia, pero no sólo de inteligencia: este modo de proceder exige la puesta en marcha de todas las facultades o posibilidades de los seres humanos que se deđican a ella. La ciencia, en efecto, exige creatividad, pensamiento autónomo, dominio de sí, capacidad para el trabajo constante y en colaboración con los demás, capacidad para confrontar socialmente el pensamiento propio con el ajeno; amor a la verdad, sensibilidad para ser afectado por problemas, intuición para percibir o inventar relaciones no comúnmente pensadas, capacidad para precisar los problemas y el lenguaje; tenacidad tanto para justificar los propios puntos de vista como para desconfiar de ellos y falsar las propias interpretaciones con metodologías adecuadas, capacidad para describir y comunicar los hallazgos de manera comprensible a la comunidad científica.

Por otra parte, el proceder cientifico posee límites internos al propio proceso y límites externos ${ }^{13}$. Internamente, toda ciencia exige, además de capacidad para la invención, capacidad para la precisión y coherencia logica, de modo que se pueda mantener, a partir de las premisas, inferencias inductivas o deductivas a largo plazo. EI proceder científico requiere igualmente la lógica de la deducción y la ruptura lógica de la invención y creación. Desce el punto de vista del hombre, esto requiere imaginación libre, creadora y también voluntad de coherencia, perseverancia en el empleo de métodos adecuados, y voluntad de justicia, de modo que el científico no esté dispuesto a beneficiar a cualquier precio a los que le pagan y dañar a los desfavorecidos con sus invenciones, sin compasión humana ${ }^{14}$.

Ahora bien el proceder científico, al ser un modo de proceder humano y social, requiere en el investigador capacidad para tratar con problemas humanos, sociales y políticos. El proceder científico no es valorativamente neutral; posee elementos de una lógica externa a su intrínseco proceso: padece presiones ideológicas de diverso tipo ${ }^{15}$.

«La llamada objetividad científica radica en la objetividad del método crítico, lo cual quiere decir, sobre todo, que no hay teoría que esté liberada de la crítica, y que los medios lógicos de los que se sirve la crítica - la categoría de la contradicción lógica- son objetivos...

„Es erróneo conjeturar que la objetividad de la ciencia depende de la objetividad del científico. $Y$ es erróneo creer que el científico de la naturaleza es más objetivo que el científico social» ${ }^{16}$.

Por otra parte, el hecho de que el proceder científico no sea neutral no es una excusa para no pretender que lo sea en el más alto grado posible; esto es, que llegue a conocer cómo son las cosas, les gusten al científico o a la comunidad científica o no les gusten los resultados a que llegan. La verdad y la objetividad, exista o no de hecho, sigue siendo no sólo una idea reguladora, sino además el ideal ético al que tiende o debería tender el proceder científico.

Precisamente porque el científico no se pone en todos los puntos de vista acerca de un problema, sino que selecciona uno o algunos, al proceder científicamente se deben explicitar los supuestos asumidos y los puntos de referencia con relación a los cuales sus afirmaciones son aceptables, y no caer en un relativismo filosofico generalizado con el que la misma comunicación científica quedaría injustificada. La responsabilidad ética de un científico le obliga a hablar con precisión y responsabilidad, estableciendo límites epistemológicos, de modo que los demás conozcan los criterios de sus afirmaciones ${ }^{17}$. La grandeza del científico se halla también en que sabe cuanto de hipotético se encuentra en sus afirmaciones, sin absolutizar, por otra parte, el valor empírico actual de la ciencia.

"En la historia de la ciencia moderna hubo teorias que fueron aceptadas por muchos científicos en un momento en el que no se disponía de confirmación experimental pura para esas suposiciones explicativas. El único fundamento para aceptarlas, en ese momento, era el hecho de que podían explicar leyes experimentales que se consideraban establecidas por datos observacionales reunidos anteriormentes ${ }^{18}$.

La creencia en la verdad y en la justicia, realizada o no, sigue siendo una de las conquistas que más nos diferencian de otras especies. El hombre, al mismo tiempo que conoce, valora lo que conoce en relación con valores extracientíficos (humanos, sociales, políticos, económi$\cos$, religiosos, etc.), que se constituyen en finalidades, para las cuales el proceder científico se convierte con frecuencia en un puro medio.

«El conocimiento científico no puede dar ningún objetivo para la vida. No establece valores válidos. Como tal no puede dirigir. La ciencia no es capaz de responder a preguntas sobre su propio sentido"l. 
«Los juicios de valor jamás pueden asumir legítimamente la forma de enunciados teoréticos, ni pueden ser puestos en relación de necesidad lógica con éstos» ${ }^{20}$.

El proceder científico tiene, pues, sus limitaciones humanas (intrigas, intereses, injusticias económicas, políticas, sociales, etc.), pero también es portador de preciados valores humanos (progreso en el bienestar individual y social, intento de objetividad y justicia, planificación del esfuerzo, utilización de la imaginación, de la voluntad, del sentimiento de colaboración, etc.). Ahora bien, la ciencia no puede ser vista como una panacea universal, pues al intentar solucionar algunos problemas provoca inevitablemente otros.

\section{INCIDENCIA DE LOS VALORES Y LIMITES DE LA CIENCIA EN UN MODELO CURRICULAR}

Varias son las instituciones educativas en las que los seres humanos se educan. La familia se ha encargado tradicionalmente de transmitir, de manera espontánea, las formas, los valores y conductas de la vida social cotidiana.

Las instituciones escolares han surgido, al hacerse la sociedad más compleja, para cubrir -entre otras- una necesidad de especialización que trascendía las posibilidades de cada familia. En particular, y en el mejor de los casos, se han propuesto posibilitar a los aprendices, de una manera sistemática, la adquisición de técnicas simbólicas (lectura, escritura, información social actualizada, metodologías, criterios de validación de los conocimientos). En las sociedades democráticas, la toma de conciencia de los diversos criterios o medidas, con las cuales se juzgan los conocimientos y conductas, ha sido una misión básica de las instituciones educativas ${ }^{21}$. En las sociedades democráticas, en las que se promueve una forma de vida autónoma, aunque dentro de la base de un bien social común, el pensar críticamente se ha convertido en una necesidad indispensable, aunque no en la única.

Sabemos que el hombre y las ciencias se mueven por intereses: intereses teóricos (saber cómo son verdaderamente las cosas) en las ciencias teóricas; intereses prácticos (saber cómo deben ser o se deben gobernar las conductas) en las ciencias prácticas; intereses de eficiencia (cómo producir mejor técnica y económicamente) en las ciencias productivas o tecnologías. Es sabido que el saber productivo sin el saber práctico y éste sin el saber teórico se vuelven inmorales: la producción no se justifíca en sí misma sino dentro de un comportamiento humano y éste dentro de un comportamiento ético. Y no hay ética sin reconocimiento de la verdad.

Ahora bien, esta exigencia es acorde con la forma de proceder del espíritu científico. Éste, en efecto, busca, como finalidad común, ante todo aunque no solamente, conocer cómo son las cosas, las causas que explican los efectos; y para esto debe aportar pruebas, que han de ser sometidas a la crítica intersubjetiva. En ciencia, las afirmaciones valen no por quien lo dice o por lo que se percibe, sino por el valor de las pruebas que se aportan.

En este contexto, la escuela significa, en gran medida: a) el paso psicológico y lógico de la percepción del mundo, b) la conceptualización y la validación del conocimiento verdadero. $\mathrm{El}$ paso de la teoría geocéntrica a la heliocéntrica significó históricamente ese trânsito: las cosas no son como se las percibe (aunque durante siglos, todos, y en todas partes han visto girar el sol alrededor de la tierra) sino el resultado de una aventura, de una creación hipotética, de un conjetura y de su provisoria validación mediante intentos đe refutación ${ }^{22}$.

Un modelo curricular implica la opción por un modelo en la forma de enseñar y aprender. Un modelo implica un conjunto sistemático de relaciones, mediante el cual, en este caso, se piensan las variables intervinientes fundamentales en el proceso de enseñar y aprender. El modelo para aprender y enseñar puede ser tomado de muy variadas fuentes: a) del modo como aprenden los animales (propuesto por los conductistas); $b$ ) del modo de proceder de los seres en la naturaleza (como lo proponía Comenio); $c$ ) del modo en que se procede en la vida social y política en general (como lo propone Freire); d) del modo como procede una computadora (en la propuesta de la teoría de sistemas); $e$ ) de modo preferentemente afectivo (como sucede en el ámbito familiar) ${ }^{23}$. Estas concepciones, aunque advierten a veces algún aspecto importante, han descuidado lo típicamente humano del modo de aprender; esto es, la reflexión sistemática sobre la experiencia y sobre los errores cometidos al buscar una solución a un problema. Porque aprender implica haber adquirido el dominio de sí, de sus posibilidades, ante el intento de solucionar problemas, no repitiendo dos veces el mismo error, ya que se refiexiona sobre la experiencia.

En nuestra concepción, la función de la escuela consiste prioritariamente en la posibilidad de aprender lo mejor posible, no en decidir por los alumnos, por las ideas o creencias que ellos defienden ${ }^{24}$. El principal objeto de estudio de los docentes debería ser el proceso de aprender y todo lo que Ileva a comprenderlo mejor. El aprendizaje humano debería tener presente el modo en que aprenden los que han procedido reflexiva y sistemática. mente como lo hacen los científicos y han aportado beneficios a la humanidad. Este modo de proceder no sólo es humano, sino que además es sistemático e implica el desarrollo armónico de todas las facultades del hombre: el sentimiento ante los problemas (intelectuales, sociales, económicos, literarios, morales, de comprensión, de aplicación, etc.), el desarrollo de la imaginación, de la voluntad, de la libertad, de la capacidad de discusión y de crítica, la capacidad de convivencia y de búsqueda del bien común, el trabajo activo y constructivo, la búsqueda de objetividad, incluso ante el propio proceso de aprender y enseñar, etc.

«La escuela debe siempre plantearse como objetivo que el joven salga de ella con una personalidad armónica, y 
no como un especialista. En mi opinión, esto es aplicable en cierto sentido, incluso a las escuelas técnicas, cuyos alumnos se dedicarán a una profesión totalmente definida. Lo primero debería ser siempre desarrollar la capacidad general para el pensamiento y el juicio independiente y no ta adquisición de conocimientos especializados. Si un individuo domina los fundamentos de su disciplina y ha aprendido a pensar y a trabajar con independencia, hallará sin duda su vía y además será mucho más hábil para adaptarse al progreso y a los cambios que el individuo cuya formación consista basicamente en la adquisición de unos conocimientos detallados» ${ }^{25}$.

Algo que se debería desterrar es la competitividad desleal, el intento del triunfo a cualquier precio. El hombre debe su fuerza en la lucha por la vida al hecho de ser un animal que vive socialmente. "El hombre que triunfa es el que recibe mucho de sus semejantes", normalmente mucho más de to que le corresponde al servicio que presta. Por eso el valor del hombre debería medirse en función de lo que da y no de lo que es capaz de recibir ${ }^{26}$.

Pensar y actuar críticamente ha sido un deseo de muchos docentes. Ahora bien, este deseo no siempre encuentra vías de realización porque falta un modelo curricular con el cual organizar y posibilitar, en la concreta situación de clase y de acuerdo con el desarrollo psicológico del que aprende, una forma sistemática y científica de evaluar los avances o retrocesos en el proceso del pensar crítico ${ }^{27}$. En este sentido, el modelo que ofrece la ciencia, por su base epistemológica, podría ser de gran ayuda para las situaciones de aprendizaje escolar. Por otra parte, este modeIo no hace más que precisar y profundizar el modo típicamente humano de aprender mediante el intento de solucionar problemas que ya se hallan en ejercicio en la vida cotidiana ${ }^{28}$.

Cualquier situación de aprendizaje (y de su facilitación mediante la enseñanza) debería comenzar por los problemas (teóricos, prácticos, tecnológicos) que afectan a los alumnos, como individuos y como sociedad, para intentar solucionarlos ${ }^{29}$.

El proceder científico aporta a la preparación y ejercicio del proceso de aprender y enseñar notables beneficios: a) une, por de pronto, la importancia de la teoría con la importancia de la práctica y la producción; $b$ ) implica el ejercicio armónico de todas las facultades humanas; c) requiere la humildad suficiente para exponer a Ios demás, y a su refutación, las más caras creencias, ideas c interpretaciones; $d$ ) exige proceder con una metodología crítica, precisa, que posibilite advertir y corregir los errores ${ }^{36}$.

Dicho brevemente, el proceder científico expresa el proceso de aprender en el más rico y complejo aspecto humano indivioual y social ${ }^{31}$. Un currículo, esto es, un instrumento para preparar la situación de enseñanza y aprendizaje, no debería ignorar los pasos fundamentales de este modo de aprender ${ }^{32}$, desde el punto de vista de la investigación, indudablemente adaptados a las circunstancias psicológicas ${ }^{3.3}$ y sociales de los alumnos y de la institución escolar ${ }^{34}$.

\section{Primera fase:}

\section{¿Qué se investigará y con qué finalidad?}

Problema y objetivo: Primera caracterización del problema. ¿Qué (hecho, dato, fenómeno) entra en conflicto, psicológico o lógico con qué (creencia, conjetura, teoría, interpretacion)?

Revisión de las opiniones o literatura existente sobre el mismo: antecedentes del problema, qué se conoce sobre ese problema, qué teorías se han formulado sobre él, con qué métodos se estudió, qué aspectos no han sido investigados.

Delimitación y definición, con terminología precisa, del problema, con lo cual queda formulado el problema (sea de literatura, de física, de historia, etc.).

Formulación de objetivos que se pretende alcanzar con la investigación; establecer claramente qué se desea investigar y para qué se investigará. ¿Qué relación tiene la investigación con los valores del hombre y de la sociedad?

\section{Segunda fase:}

\section{¿Cuál es la base teórica del problema?}

Marco teórico: Sugerencias, ideas conductoras, conceptos, definiciones, hipótesis; elaboración explícita de la teoría que se sustenta.

Prueba, a partir del principio de no-contradición, de que la explicación teorica elaborada es coherente.

(Hasta aquí, todos Ios pasos del proceso de investigación son igualmente válidos para una ciencia formal como para una ciencia empírica).

Paso de las variables conceptuales a variables operacionales (si se trata de un problema que será empíricamente investigado). Por ejemplo: pasar del concepto de pobreza a establecer cómo (con qué parámetro) se conprobará de hecho, empíricamente, que una determinada persona es pobre.

\section{Tercera fase:}

¿Cómo se investigará?

Disef̃o metodológico de la fase empírica de la investigacion:

Población y muestra que se seleccionará para estudiar la hipótesis realizada para comprender el problema. 


\begin{tabular}{|c|c|c|c|}
\hline & Ámbito cotidiano & Ámbito científico & Didáctica científica \\
\hline $\begin{array}{l}\text { Objetivos } \\
\text { Subobjetivos }\end{array}$ & $\begin{array}{l}\text { - Llevar una buena vida. } \\
\text { - Explicaciones } \\
\text { inmediatas. } \\
\text { - Poca preocupacipon por la } \\
\text { coherencia. }\end{array}$ & $\begin{array}{l}\text { - Finalidad instrumental. } \\
\text { - Precisión y explicación } \\
\text { optimas y mediatas. } \\
\text { - Máxima preocupación } \\
\text { por la coherencia. }\end{array}$ & $\begin{array}{l}\text { - Educar. } \\
\text { - Enscriar: posibilitar } \\
\text { aprender comprendiendo } \\
\text { con precisión (en forma } \\
\text { adecuada a cada alumno), } \\
\text { formas de saber y de vivir. }\end{array}$ \\
\hline \multicolumn{4}{|l|}{ Objetivos de trabajo } \\
\hline b) Evaluacion de la valicez. & $\begin{array}{l}\text { a) - Pocas inferencias. } \\
\text { - Varias premisas } \\
\text { aceptables. } \\
\text { b) - Evaluar tiene una } \\
\text { importancia moderada. } \\
\text { - Evaluar observaciones e } \\
\text { inferencias plausibles. } \\
\\
\text { c) - Interés para la vida. }\end{array}$ & $\begin{array}{l}\text { a) - Muchas inferencias. } \\
\text { - Premisas bien } \\
\text { especificadas. } \\
\text { b) - Evaluar tiene una } \\
\text { importancia fundamental. } \\
\text { - Evaluar datos, inferencias } \\
\text { especificas y la metodologia } \\
\text { (epistemologia). } \\
\\
\text { c) - Intereses teóricos, } \\
\text { prácticos o productivos. }\end{array}$ & $\begin{array}{l}\text { a) - Posibilitar el aprendizaje } \\
\text { significativo y comprensivo. } \\
\\
\text { b) - Evaluar con precisión la } \\
\text { comprensión y dominio } \\
\text { adecuado a cada alumno y a } \\
\text { cada forma de saber y hacer. } \\
\text { - Evaluar el marco térico y } \\
\text { los resultados empiricos del } \\
\text { modo de enseñar. } \\
\text { c) - Interés formativo. }\end{array}$ \\
\hline \multicolumn{4}{|l|}{ Cognición del ámbito } \\
\hline - Conceptos & - Prácticos, imprecisos. & - Explicitos, exactos. & - Posibilitar construirios. \\
\hline - Organización & - Asociación, con coherencia local. & $\begin{array}{l}\text { - Organización lógica y } \\
\text { epistemológica, giobaimente } \\
\text { coherente. }\end{array}$ & $\begin{array}{l}\text { - Aprender según las } \\
\text { posibilidades psicológicas y } \\
\text { logico-epistemológicas } \\
\text { de cada saber. }\end{array}$ \\
\hline - Métodos & $\begin{array}{l}\text { - Resolución informal y práctica de } \\
\text { problemas. } \\
\text { Breves inferencias. }\end{array}$ & $\begin{array}{l}\text { - Resolución fomal, } \\
\text { teórico-práctica, con } \\
\text { técnicas y largas inferencias. }\end{array}$ & $\begin{array}{l}\text { - Posibilidad de resolver } \\
\text { problemas según el método de } \\
\text { cada saber específico y las } \\
\text { capacidades psicológicas de } \\
\text { cada alumno. }\end{array}$ \\
\hline \multicolumn{4}{|l|}{ Preocupación por la calidad } \\
\hline - Control de calidad & - Informal, sin método. & - Estricta, explícita. & $\begin{array}{l}\text { - Posibilita controlar con } \\
\text { precisión el enseñar y el } \\
\text { aprender, y evaluar } \\
\text { éticamente. }\end{array}$ \\
\hline - Eficacia & - Eficiente en tareas cotidianas. & - Eficiente en tareas complejas. & $\begin{array}{l}\text { - Eficiencia limitada, } \\
\text { compartida con la tarea del } \\
\text { alumno y la institución. }\end{array}$ \\
\hline Valores & $\begin{array}{l}\text { - Vitales, inmediatos o de corto } \\
\text { plazo. }\end{array}$ & $\begin{array}{l}\text { - Valor de instrumento de } \\
\text { investigacion para diversos fines } \\
\text { que escapan a veces a la } \\
\text { decisión del científico. }\end{array}$ & $\begin{array}{l}\text { - Explícizos en el concepto de } \\
\text { educación, a los que sirve la } \\
\text { didáctica como instrumento. }\end{array}$ \\
\hline
\end{tabular}

Tipo de estudio empírico, observacional o experimental que realizará: por ejemplo, qué método de recolección de datos empleará, con qué procedimien- tos (entrevista, encuesta, etc.). Consulta o trabajo en equipo con especialistas (psicólogos, sociólogos, etc.), según el caso. 
Como trabajará con los datos o hechos relevantes: plan de tabulación, de análisis estadístico, etc.

\section{Cuarta fase:}

\section{Ejecución}

Elaboración teórica (si se trata de una investigación teórica) o elaboración empírica:

Recolección de la información o de los datos.

Presentación de la misma.

Utilización de las hipótesis o teoría para el análisis de la información o datos.

Conclusiones y recomendaciones.

Redacción final đel trabajo: informe, artículo, libro, etc.

Indudablemente que no todos los aprendices se hallarán en condiciones psicoevolutivas de realizar estos pasos; pero incluso en niños muy pequeños se halla la curiosidad, el deseo de investigar, que constituye lo esencial del proceso científico. Esto puede cultivarse desde muy temprana edad.

Sobre esta base cientifica, controlable, se puede entender, de forma más concreta, qué significa pensar, aprender constructivamente intentando solucionar problemas $^{35}$, aprender reflexivamente ${ }^{36}$. Y sobre esta base es posible realizar una evaluación pautada y objetivada, tanto sobre los procesos fundamentales del pensamiento científico que aprende el alumno (observar en determinada forma, con precision, interpretar creativamente, realizar inferencias, aplicar a situaciones nuevas, refutar las creencias o conjeturas, etc.) como sobre los resultados alcanzados. En efecto, cuando, al docente, le faita una teoría didáctica expresa, la evaluación no puede sino basarse, en el mejor de los casos, en el sentido común, en el más o menos, o en una intuición sincrética y sibilina del docente ${ }^{37}$.

Está claro que una institución educativa tiene la función de ayudar, dentro de sus posibilidades, a aprender de la forma más adecuada a cada alumno, y no puede identificarse, sin más una escuela a un laboratorio de investigación donde la intencionalidad docente suele estar ausente. Por otra parte, un niño, si bien tiene inquietudes de investigación y curiosidad, no puede considerarse todavia un investigador adulto.

Hay que reconocer, además, que el ámbito de la expresión desinhibida no se rige por normas y no se atiene al proceder de ningún modelo científico; pero, en la medida en que esa expresion se hace arte, se construye, se transmite y se socializa, comienza a requerirlas. No hablamos, pues, de un currículo para la expresión artistica, sino para todo aquello que implique aprender sistemática y reflexivamente.

Ahora bien, concebimos las instituciones educativas como lugares no sólo de expresión libre de la personalidad, sino además y principalmente como lugares en que es posible el aprendizaje sistematizado. Este aprendizaje exige un currículo $\mathfrak{u}$ organización de las actividades tanto a nivel de institución como a nivel del concreto proceso de enseñanza y aprendizaje ${ }^{38}$.

Un currículo exige una teoría curricular y una modelización científica de esa teoría. Esto incluye asumir una teoría del conocimiento humano y científico, o sea una base epistemológica. Pero dentro del ámbito de la epistemología existen diversas teorías epistemológicas que difieren sobre todo por los criterios que asumen para validar los conocimientos científicos.

\section{El empirismo exige criterios empíricos.}

El racionalismo demanda criterios racionales (la coherencia, la no-contradicción, la sistematicidad y la mayor simplicidad posible).

La epistemología de Popper exige la falsabilidad de los enunciados.

La epistemología de Lakatos estima científico todo programa de investigación que tiene capacidad heurística.

La epistemologia de Kuhn postula paradigmas socialmente compartidos por los científicos para realizar una ciencia normal.

La epistemología de Laudan requiere que los conocimientos, para que sean considerados científicos, se atengan a las tradiciones de investigación.

La epistemología anarquista de Feyerabend sostiene que todo vale en ciencia ${ }^{39}$.

Un currículo exige, además de una base epistemologica clara (saber qué epistemología se asume, qué se va a enseñar, cuál es su estructura cientffica, sus procesos típicos), conocer las posibilidades personales o psicologicas de quienes aprenden (que creencias, evidencias, conceptos previos poseen sobre el asunto a tratar, qué posibilidades de abstracción y dominio simbólico manejan, etc.) y conocer qué posibilidades de recursos didácticos materiales posee la institución ${ }^{40}$.

Dicho brevemente, una ciencia al entrar en la institución educativa debe ser convertida por el docente en disciplina (discere: aprender), esto es, aprendible para la situación concreta de los alumnos.

Al enseñar y aprender, según el espíritu de la ciencia, que es crítico por su misma naturaleza, el docente no puede caer en la contradicción đe enseñarla dogmáticamente ${ }^{41}$. Al aprender, cada uno construye su saber sobre problemas reales. Nadie aprence por otro, aunque todos necesitemos ayuda y solidaridad en ese proceso.

\section{A MODO DE CONCLUSIÓN}

La investigación científica no es el invento de un modo de proceder propio de la modernidad, sino de la humani- 
dad. El modo científico de proceder prolonga una forma típicamente humana de proceder. En efecto, en ese modo de proceder afloran valores y derechos irrenunciablemente humanos como la capacidad de dudar, Ia creencia en la verdad, la creencia en la racionalidad sin absolutizarla, sin reducir los fines a los medios, la capacidad para ser afectado por los problemas individuales y sociales, el valor de su búsqueda, la libertad para investigarla, la autonomía intelectual, Ia igualdad de derecho para la discusión sin privilegios para la autoridad o la fuerza física, el valor del diấlogo en la investigación y validación de los conocimientos y de las conductas individuales y sociales ${ }^{42}$.

En una época posmoderna, en donde los feelings es lo que cuenta, la combinación de la imaginación con la lógica de la investigación ayuda a ordenar los comportamientos ${ }^{43}$. Estimamos que son didácticas parciales aquélias que, sobre todo en los niveles de la escuela general básica o incluso en los segundos niveles de educación, acentúan sólo algunos aspectos de las múltiples posibilidades del hombre, atrofiando o minusvalorando otras. Una educación integral implica, como instrumento, la utilización de un modelo curricular en el que tengan cabida distintas formas sociales de saber (ciencias, disciplinas, visiones del mundo), pero además un modelo curricular en el que sea posible el cultivo de las competencias y facultades múltiples del ser humano. Ahora bien, una concepción amplia, análoga del proceder científico, da elementos para posibilitar a todo el ser humano, porque han sido los seres humanos (los grandes hombres de la humanidad) los que con sentimiento de admiración, con esfuerzo metódico de todo tipo, con inteligencia e interacción social en búsqueda de validaciones, han creado la estructura del proceder científico.
Por otra parte, el docente, al elegir una didáctica con base epistemológica, debe ser consciente de qué epistemología elige, pues no se trata de una opción neutra ya que conlleva la opción por un tipo de hombre, de sociedad y de educación. Si no somos conscientes de ello, estaremos cayendo nuevamente en manos de un currículo oculto ${ }^{44}$.

El docente, de acuerdo con nuestra propuesta, en el momento de preparar el currículo como modelo de instrumento didáctico debería tener presente: a) el $a m b i$ to de la cotidianeidad, con los conceptos y mentalidades previas que asumen los que ingresan a un estudio sistemático como debería ser el escolar; b) que ese ámbito psicológico y social puede ser enriquecido, de forma adecuada a nuestra cultura científica y técnica, utilizando un currículo de base epistemológica aplicada al saber que facilita aprender; c) como consecuencia, su didáctica tendrá en cuenta no sólo que sus conocimientos, en cuanto a contenidos curriculares, se hallen actualizados, sino además que esos conocimientos específicos sean construidos, por quien aprende con acciones teóricas y prácticas, con procedimientos y actitudes individuales, sociales y éticas, adecuadas al modo como to hace el científico de esa especialidad; $d$ ) sin pretender hacer de cada alumno un científico en miniatura, la estructura epistemológica de cada saber específico sirva de pauta objetiva en la interacción subjetiva del docente y del alumno, al tiempo que sugiera el desarrollo de aspectos humanos no solamente ceñidos al conocimiento, sino además a la voluntad, a la libertad de pensamiento y de interacción social para validar los conocimientos, al respeto mutuo a Ias propias hipótesis y creencias, al reconocimiento de los propios límites y de los propios logros.

\section{NOTAS}

1 ARISTÓTELES. Segundos analíticos, L. I, cap. 2. Para los límites y valores de esta concepción, véase: NAGEL, E. (1978). La estructura de la ciencia, p. 51. Buenos Aires: Paidós.

2 FEYERABEND, P.(1974). Contra elmétodo, p. 134. Barcelona: Ariel.

3 DARÓS, W. (1984). La analogía en el concepto de ciencia aristotélico-tomista. Sapientia, Vol. 39, pp. 19-36. DAROS, W. (1984), Razóne inteligencia. (Centro Editoriale di Cultura, Génova). DE ALEJANDRO, J. (1984). Gnoseologia, p. 480. Madrid: BAC.

4 POPPER, K. (1977), La lógica de la investigación científica, p. 51. Madrid: Tecnos.

5 FEYERABEND, P., Contra el método, p. 134. Pardo, R. (1972). La ciencia y la filosofia como saber sin ser, p. 215 . (Universidad, Rosario). DAROS, W. (1980). Racionalidad, ciencia y relativismo, p. 131. Rosario: Apis.

6 EINSTEIN, A. (1981). Misideasyopiniones, p.61. Barcelona: Bosch.

, EINSTEIN, A. (1981). pp. 291-292. KLIMOVSKY, G. Ciencia e ideologia, en Sabato, G. (comp.) (1975). El pensamiento latinoamericano en la problemática cienciatecnología-desarrollo-dependencia, p. 23. Buenos Aires: Paidós.

8 POPPER, K. (1984). Conocimiento objetivo. Un enfoque evolutivo, p. 181. Madrid: Tecnos. 
9 EINSTEIN, A. y INFELD, L. (1974). La fisica, aventura del pensamiento, p. 250. Buenos Aires: Paidos. ORTEGA y GASSET, J. (1983). Obras completas, Vol. V, p. 404. Madrid: Alianza.

10 POPPER, K. (1970). Sobre la teoria de la inteligencia objetiva, en Simposio de Burgos. Ensayos de Filosofía de la Ciencia, p. 232. Madrid: Tecnos.

" POPPER, K. (1977). La logica de la investigación cientifica, p. 106. Madrid: Tecnos. Según G. Bachelard, «el espiritu puede cambiar de metafisica, pero no puede pasarse sin ella». BACHELARD, G. (1973). Epistemología, p. 27. Barcelona: Anagrama.

12 MARTÍNEZ, J. (1980). Ciencia y dogmatismo. El problema de la objetividad en Karl R. Popper. Madrid: Cátedra. SERRANO, J.(1991). La objetividad y las ciencias. Enfoque histórico-epistemológico. México: Trilla.

13 KUHN, Th. (1988). La tensión esencial. Estudios selectos sobre la tradición y el cambio en el ámbito de la ciencia, pp. 2 I y 143. México: FCE. KUHN, Th. (1979). La función del dogma en la investigación científica, p. 4. Vatencia: Cuadernos Teorema. BUTTERFIELD, H. (1982). Losorígenes de la ciencia moderna, p. 9. Madrid: Taurus. BERNAL, I. (1979). Laciencia en nuestro tiempo. México: Nueva Imagen. HOROWITZ, I. (1968). Historia yelementos de la sociología del conocimiento. Buenos Aires: Audeba. HICKS, D. y POTTER, J. (1991). Sociology of Scientific Knowledge. Social Studies of Science, Vol. 21, p. 459-501.

14 LOPE7, A. et al. (1992). The Development of Categorybased Induction. Child Development, Vol. 63(5), pp. 1070 1091. LAKATOS, I. (1988). Pruebas y refutaciones. La lógica del descubrimiento matemático. Madrid: Alianza. SANTALO, L. (1976). Geometrias no euclidianas. Buenos Aires: Eudeba. GÓMEZ, R. (1977), Las teorias cientificas. Desarrollo, estructura, fundamentación. Buenos Aires: Coloquio. SCHLANGER, J. (1983). L'invention intellectuelle. Paris: Fayard. OSBORN, A. (1984). L'imagination constructive. Paris: Dunod. WALLACE, W. (1986). La lógica de la ciencia enla sociología. Madrid: Alianza. BOURDIE, P. et al. (1989). El oficio de sociologo. Buenos Aires: Siglo XXI.

15 SUÁREZ, F. et al (1985). Autonomía nacional o depentdencia: la politica cinetífico-tecnológica. Buenos Aires: Paidos. BOUDON, R. (1980). Efectos perversos y orden social, p. 79. México: Premia. PALMADE, G. (1988). Interdisciplinariedade ideologías. Madrid: Narcea. REBOUL, O. (1977). L'endoctrinement. París: Puf.

16 POPPER, K. (1978). La lógica de las ciencias sociales, pp. 12 y 17. México: Grijalbo. HEISENBERG, W. (1976). La imagen de la naturaleza en la física actual, p. 12-13. Barcelona: Ariel.

17 LAKATOS, 1. (1975). La historia de la ciencia y sus reconstrucciones racionales, en Lakatos, I. y Musgrave, A. La crítica yel desarrollo del conocimiento, p. 473. Barcelona: Grijalbo.

18 NAGEL, E. (1978). Estructura de la ciencia, p. 90. Buenos Aires: Paidós. POPPER, K. La logica de la investigación cientifica, p. 57.

19 JASPER, K (1985). Filosofia de la existencia, p. 17. Barcelona: Barcelona.

20 HABERMAS, I. (1978). Teoría analítica de la ciencia y dialéctica, en La lógica de las ciencias sociales, p. 69. México: Grijalbo.
2) ARROYO GARRIDO, S. (1992). Teoría y prúctica de ka escuela actual. Madrid: Siglo XXI. GRUWE7. J. (1992). Le projet d'école. I.jlle: CRIP. FRABBONI, F. y GENOVESI, G. (1990). La scuola e i suoi problemi. Per una teoria della scuola. Firenze: La Nuova Italia.

22 DARÓS, W. (1984). El concepto de revolución científica en Razón elnteligiencia, pp. 85-126. Génova: Centro Editoriale di Cultura.

${ }^{23}$ CHADWICK, C. (1989). Teorías del aprendizaje para el docente. Santiago de Chile: Universitaria. CARR, W, y KEMMIS, ST. (1988). Teoría crítica de la enseñana. Barcelona: Roca. DARÓS, W. (1987). Diversas bases para una teoría didáctica. Revista de Ciencias de la Educación, 130, pp. 215-225. DAROS, W. (1986). La educación o lá conducta cientifica y heterónoma del hombre según el pensamiento de Skinner, B. Revista Española de Pedayogia, 173, pp. 341-356. EISNER, E. y VALLANCE, E. (1987). Cinco concepciones del curriculum. Dicac, 11, p. 19

${ }^{24}$ ASTOLFI, J. P. (1992). Lécole pour apprendre. París: FSF. VECCHI, G. (1992). Aider les élèves à apprendre. París: Hachette. STEINDORF, G. (1991), Grundbegriffe des lehren. und Lernens. Bad Heilbrunn: Klinkhardt.

${ }^{25}$ EINSTEIN, A. Mis ideas y opiniones, p. 56.

26 Ídem, p. 55 .

27 ZABALZA, M. (1991). Diseño y desarrollo curriculur. Madrid: Narcea. FELDMAN, D. (1993). ¿Qué prácticas, qué teorías? Algunas precisiones conceptuales para el estudio de la "práctica docente". Revista Argentina de Educación, 20 , pp. $27-37$

${ }^{28}$ WAGNER, B. y PHILI.IPS, D. (1992). Beyond Beliefs: Parent and Child Behaviors and Children's Perceived Academic Competence. Child Development, Vol. 63(6), pp. $1380-1392$.

2 COLL, C. (1988). Significado y sentido en el aprendizaje escolar. Reflexiones en torno al concepto de aprendizaje significativo. Infancia y Aprendizaje, 41, pp. 131-142.

30 PALACIOS, C. y ZAMBRANO, E. (1993). Aprender y enseñar ciencias: una relación a tener en cuenta. Boletín. Proyecto principal de educación en América latina y al Caribe. Santiago de Chile, UNESCO, Orealc, n. 31, pp. 5069. MORENO, M. (1983). La pedagogía operatoria. Enstañunzu de las ciencias, 4(1), pp. 57-63.

31 SANTALÓ, L. (1980). Matemática y sociedad. Por uhé y para qué enseñar matemática en la Escuela. Buenos Aires: Docencia. GOZZER, G. (1972). La educación tecnológica. Buenos Aires: Ateneo.

32 MELLADOV, V y CARRRACEIO, D. (1993). Contribuciones de la filosofía de la ciencia a la didáctica de las ciencias. Enseñanza de las ciencias, 11(3), pp. 331-341. CANAI.FS,

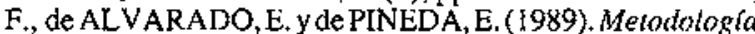
de la investigación. Manual para el desarrollo de personal de salud. (Organización Mundial y Panamericana de la Salud, Washington) p.60. DEWEY, J. (1989). Cómo pensamess. Nueva exposición de la relación entre pensamiento reflexivo y proceso educativo, pp. 102-103. Barcelona: Paidós.

3 ESPINOSA-GARCÍA, J. y ROMÁN GALÁN, T. (1993). Actitudes hacia la ciencia en estudiantes universitarios do ciencias. Enseñanza de las ciencias, 1](3), pp. 289-297. POZO, J. et al. (1991). Las ideas de los alumnos sobre la ciencia. Enseñanza de las ciencias, 9(1),pp. 83-95. HARRIS P. et al. (1994). Young Children's Comprehension. Child Development, 1, pp. 16-30. VIDAL-ABARCA, E. y 
GILABERT, R. (1994). Mapas de ideas: una herramienta para el aprendizaje escolar. Datos y comentarios para una discusión. Comunicación, Lenguaje y Educación, 21, pp. 75-86. HERNÁNDEZ HERNANDEZ, P. y GARCÍA, L. (1991). Psicología yenseñanza del estudio. Teorias y técnicas parapotenciarlas habilidades intelectuales. Madrid: Pirámide.

34 REIF, F. y LARKIN, J. (1994). El conocimiento científico y el cotidiano: comparación e implicaciones para el aprendizaje. Comunicación, Lenguaje y Educación, 2I, pp. 3-30.

${ }^{35}$ DESCAVES, A. (1992). Comprendre des énoncés, résoudres des problémes. París: Hachette. DE LORENZO, G. (1992). Question de savoir Introduction àune méthode de construction autonome des saboir. París, ESF, 1992. CONTRERAS DOMINGO,J. (1991). El sentido educativo de la investigación. Cutadernos de Pedagogia, 196, pp. 61-67. STENHOUSE, L. (1991). Investigación y desarrollo del curriculum. Madrid: Morata. PARRA MOSQUEDA, B. (1989). Acerca deI papel de la representación en la resolución de problemas. Pedagogía, 17, pp. 33-40.

36 DAROS, W. (1992). Teoria del aprendizaje reflexivo, pp. 225-226. Universidad Nacional de Rosario: CONICET.

${ }^{77}$ REIF, F. y LARKIN, J. El conocimiento cientifico y el cotidiano: comparación e implicaciones para el aprendizaje, pp. 24-25. DARÓS, W. (1981). Encuadre episte-mológico de la didáctića experimental. Didáctica Experimental, $1, \mathrm{pp}$. 31-64. DARÓS, W. (1983). El concepto filosóficode «ciencia» según Popper. Rivista Rosminiana, F. III, pp. 257-271.

38 DARÓS, W. (1993). Construcción de los conocimientos y diseño curricular de base epistemológica. Revista IRICE, 7 , pp. 5-55.

39 MELLADO, V. y CARRACEDO, D. (1993). Contribución de la filosofía de la ciencia a la didáctica de las ciencias. Enseñanza de las ciencias, 11(3), pp. 331-339. NIAZ, M. (1994). Más allá del positivismo: una interpretación lakatosiana de la enseñanza de las ciencias. Enseñanza de las ciencias, 12 (1), pp. 97-160. DARós, W. (1992). Función de las teorías en educación. Teoria del aprendizaje reflexivo, pp. 9-46. BENSON, G. (1989). Epistemology and Science Curriculum. Journal of Curriculum Studies, 21(4), pp. 329344. ESTANY, A. (1990). Modelos de cambio científico. Barcelona: Crítica. LOPEZ RUPÉREZ, F. (1990). Epistemología y dióáctica de las ciencias. Enseñanza de las ciencias, 8(1), pp. 65-74. PORLAN, R. (1990). Hacia una fundamentación epistemologica de la enseñanza. Investigación en la Escuela, 10, pp. 3-32.
40 GRUNDY, S. (1991). Producto o praxis del curriculum. Madrid: Crítica. KEMMIS, S. (1988). El curriculum: más allá de la teoria de la reproducción. Madrid: Morata. TORRES, J. (1992). El curriculum oculto. Madrid: Morata. ZABALZA, M. (1991). Diseño y desarrollo curricular. Madrid: Narcea. FELDMAN, D. (1993). ¿Qué prácticas, qué teorias? Algunas precisiones conceptuales para el estudio de la "prắctica docente». Revista Argentina de Educación, 20, pp. 27-37. BURBULES, N. y LINN, M. (1991). Science Education and Philosophy of Science: Congruence or Contradiction? International Journal of Science Education, $13(3)$, pp. 227-241.

4I LINZ, L (1983). EL efecto liberador de la ciencia en la vida social. BENZO, C. et al. (1983). Once ensayos sobre la ciencia, p. 219. Madrid: Iuan March. MENDOZACARRERA, E. (1993). La construcción del conocimiento en la investigación sobre la enserianza de la ciencia. Perfiles Educativos, $60 \mathrm{p}$. 73. WENHAM, M. (1993). The nature and role of hypothesis in school science investigations. International Journal of Science Education, 3, p. 231 -240. LEGRAND, J. L. (1992). Epistémologie et implications: vers une heuristique implicationnelle. Les Sciences de l'Éducation, S, p. 61-75. RUIZ, B. (1993). Aprendizaje por descubrimiento: principios y aplicaciones inadecuadas. Enseñanza de las Ciencias, 11(1), pp. 3-11.

42 TOUMEY, C. (1991). Modern Creationism and Scientific Authority. Social Studies of Science, 21(4), pp. 621-699. FINCHER, C. (1991). The possibilities and actualities of disciplined inquiry. Researh in Higher Education,32(6), pp. 625-650.

${ }^{43}$ DARÓS, W. (1994). Fundamentos antropológico-sociales de la Educación, pp. 190-199. Villa Libertador San Martín: UAP. OBIOLS, G. (1993). Adolescencia, posmodernidady escuela secundaria, p. 15. Buenos Aires: Kapelusz. LYOTARD, J.F. (1987). La condición postmoderna. Buenos Aires: REI. LIPOVETZKY, G. (1986). La era del vacio. Ensayos sobre el individualismo contemporáneo. Barcelona: Anagrama. FINKIELKRAUT, A., (1990). La derrota del pensamiento. Barcelona: Anagrama.

44 TORRES, J. (1992). El curriculum oculto. Madrid: Morata. GRUNDY, S., 1991. Producto y praxis del curriculum, p. 19. Madrid: Morata. JANK, W. y MEYER, H. (I991). Didaktische modelle. Frankfurt: Cornelsen Scriptor. GRASSILLI, B. (I992). Per una teoria didattica. Pedagogia e Vita, 6, pp. 66-82. VERTECCHI, B. (1990). Interpretazioni della didattica. Firenze: La Nuova Itatia. 
